\title{
NISTIR 5673
}

\section{Information Technologies Make Business Sense for the Custom Therapeutic Footwear Industry}

Howard T. Moncarz

U.S. DEPARTMENT OF COMMERCE

Technology Administration

National Institute of Standards

and Technology

Manufacturing Systems Integration Division

Gaithersburg, MD 20899

QC

100

.056

NLT

N0.5673

1995 



\title{
Information Technologies Make Business Sense for the Custom Therapeutic Footwear Industry
}

\author{
Howard T. Moncarz
}

U.S. DEPARTMENT OF COMMERCE

Technology Administration

National Ins titute of Standards

and Technology

Manufacturing Systems Integration Division

Gaithersburg, MD 20899

June 1995

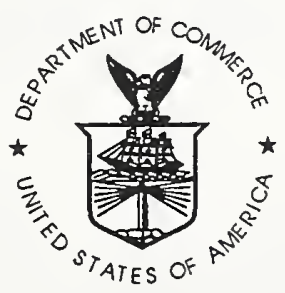

U.S. DEPARTMENT OF COMMERCE Ronald H. Brown, Secretary

TECHNOLOGY ADMINISTRATION

Mary L. Good, Under Secretary for Technology

NATIONAL INSTITUTE OF STANDARDS

AND TECHNOLOGY

Arati Prabhakar, Director 



\title{
Information Technologies Make Business Sense for the Custom Therapeutic Footwear Industry
}

\author{
Howard T. Moncarz \\ National Institute of Standards and Technology
}

\begin{abstract}
The commercialization and integration of emerging technologies for custom therapeutic footwear (CTF) manufacturing will provide economic and health-care benefits to the nation and will improve responsiveness to consumer desires. "Commerce At Light Speed" (CALS) technologies, in particular the Standard for the Exchange of Product Model Data (STEP), can greatly increase the benefits that are possible. Using STEP Application Protocols (APs), complex product information can be unambiguously transferred throughout the virtual and distributed, multi-enterprise marketplace that is anticipated for the future CTF industry. In that marketplace, information representing foot shapes, shoe lasts (forms for footwear manufacturing), orthopedic inserts, and the patterns that comprise the footwear pieces must be transferred among different organizations. Those organizations will be geographically distributed, involved in different phases of the CTF manufacturing life cycle, and all connected through the virtual marketplace. This paper will describe that marketplace, the type of STEP APs required, and possible business scenarios.
\end{abstract}

\section{1: Introduction 1}

Although the current U.S. market for custom therapeutic footwear (CTF) is fewer than 100,000 pairs of shoes a year, medical experts estimate that over 10,000,000 Americans could benefit from CTF. ${ }^{2}$ Furthermore, it is estimated that $\$ 1.5$ billion could be saved annually by the prevention of 45,000 (out of 54,000 ) foot amputations of diabetics. Another $\$ 3.5$ billion could be saved annually by avoiding surgery for common foot problems that women develop as a result of wearing poorly fitting shoes. Finally, the

\footnotetext{
${ }^{1}$ The data presented in this paper was provided largely by the participants at a workshop held in Charleston, South Carolina in March 1994, including subsequent discussions with them [1]. The participants were leaders from the mainstream and custom therapeutic footwear industry, and also included representatives from the medical, academic, government, and military communities.

2 Note that the terms footwear and shoes are used interchangeably in this paper.
}

possibility of providing custom, comfortable-fitting, and stylish shoes at an affordable price with wide distribution could create a large, new market that the American footwear industry could exploit.

In fact, the U.S. domestic footwear market is $\$ 32$ billion a year at retail, which translates to over 1.5 billion pairs of shoes sold annually. Just 25 years ago, $80 \%$ of the shoes bought in this country were manufactured here. Now, however, that $80 \%$ figure has shrunk to only $12 \%$, with the number of domestic shoe manufacturers likewise reduced. The capability and infrastructure to provide affordable and stylish, custom footwear has promise not only to improve Americans' health but also to strengthen the mainstream, American-footwear industry.

For manufacturers to fully supply the potentially huge custom and CTF market, complex product information must be unambiguously transferred throughout a distributed and virtual, multi-enterprise marketplace. Each process throughout the manufacturing life cycle of the product requires complex information, produces new complex information, and may quite realistically be done by different individuals or organizations that are geographically distributed. The Standard for the Exchange of Product Model Data (STEP) is well suited to represent the product information necessary to enable the effective communication of product information throughout the CTF virtual enterprise.

Although standards for product information are critical enablers to the CTF virtual enterprise and are the subject of this paper, it should be noted that other $\mathrm{CALS}^{3}$ technologies and standards are also necessary to create the CTF information infrastructure. Those other technologies and standards are associated with business transactions, interface management, work-

\footnotetext{
${ }^{3}$ The acronym, CALS, as used by the Department of Defense, stands for "Continuous Acquisition and LifeCycle Support." MG James R. Klugh (USA, Ret), Deputy Under Secretary of Defense (Logistics) has recently offered a new definition for CALS, "CALS is a CORE strategy to use integrated data through a set of standards to achieve efficiencies in business and operational mission areas of the Department of Defense." However, CALS is now popularly known as "Commerce At Light Speed," and is applied to any type of business enterprise.
} 
flow management, distributed data/information management, etc.

To help develop the necessary standards, the National Institute of Standards and Technology (NIST) initiated a collaborative research and development program in October 1994 to advance the use of information technology in the custom therapeutic footwear (CTF) industry. The main technical goal of NIST in this project is to help develop STEP Application Protocols (APs) to support the CTF life cycle. (A STEP AP extends the standard to specific interfaces.) A second goal of NIST is to act as a catalyst to enable the footwear industry to develop or utilize the other manufacturing technologies needed, including those necessary to complete the information infrastructure described above.

The first step necessary is an understanding of the CTF manufacturing life cycle. That is the subject of the next section.

\section{2: Manufacturing Life Cycle}

The CTF manufacturing life cycle can be separated into sequential phases that are similar to those of other industries:

- Requirements-the customer's product requirements must be determined;

- Design-a product is specified which meets the requirements;

- Manufacturing Engineering-a production plan is created to make the product to best meet the company's production capability;

- Production-the product is produced;

- Distribution-the product is packaged and shipped to the customer;

- Service-customer service closes the loop to ensure that the product meets the customer's expectations, and should reflect the initial requirements. (If they do not, new requirements are necessary.)

The general processes required for CTF manufacturing apply whether the processes are done manually or whether they are automated using advanced technology. In this section, the processes for making CTF are described in a general way without referencing particular methods or technologies. The CTF processes and an idea of what type of company, organization, or individual will execute each process in the future is shown in Figure 1: Custom Therapeutic Footwear Manufacturing Life Cycle.

\section{1: Requirements}

Three types of specifications are required for CTF. First of all, the customer who has a foot problem goes to a medical professional (for example, a podiatrist) who examines and diagnoses the problem. If appropriate, the podiatrist provides the customer a prescription for how custom footwear must be adapted to accommodate and/or treat the problem. Next, the customer goes to a pedorthist, a professional with special expertise to provide CTF. The pedorthist will measure the feet to provide the second specification required. Finally, the customer chooses the footwear style and color desired, the third specification. The style choices provided are from a selection of appropriate styles that can accommodate the particular customer's fit and medical needs.

\section{2: Design}

"The last comes first." [2] The last is a 3D shoe form of wood, plastic, or other material used in the shoe manufacture. The last captures the style of the shoe desired for a specific sized and shaped foot. Once the last has been designed, the footwear itself (including all of the footwear pieces) and a removable orthopedic insert, if necessary, can be designed. The orthopedic insert will fit in the bottom of the shoe, matches the foot bottom, and adds accommodative/therapeutic corrections as required for maximum comfort and benefit. Both the footwear and the orthopedic insert designs use the last design as input of the shoe form that is required.

\section{3: Manufacturing Engineering}

Given the design specifications, the footwear manufacture can now be planned for the processes needed to make and assemble each component required, and matched to the specific facility's production equipment. It is likely that the company that will manufacture the last will also plan its manufacture; the situation is similar for the footwear itself and the orthopedic insert.

The main task to plan the last and insert manufacture is to create the toolpaths or files needed for numerically-controlled (NC) manufacturing or rapid prototyping (e.g., stereolithography using STLformatted files) of the respective 3D shapes. Some assembly of the separately manufactured components will be necessary. The last is generally composed of at least two main pieces that are hinged together, so that the last can be bent and removed at the end of the 
footwear manufacture. The insert is often composed of layers that must be bonded together.

The footwear consists of the upper, assembled from a number of pattern pieces; the bottom, which is the sole and the heel; the insole which is the interface between the upper and the bottom; and the accessories required, such as tassels, eyelets, shoelaces, etc. The material of choice, particularly for CTF, is leather. Leather is typically nonhomogeneous. Some areas of the leather cannot be used at all because of defects; other areas have varying levels of quality (i.e. varying grade) and need to be intelligently matched to the specific footwear pattern pieces required. For example, the vamp, which is the main pattern piece on the top front of the shoe should use the best quality leather available. from them by the footwear manufacturer. It is not unlikely that the last, the insert, and the footwear will be made by different organizations throughout the country. The footwear manufacturer will likely make the "upper" of the shoe, possibly the heel and sole, and finally manufacture the shoe, using the last as a form in the manufacturing process.

\section{5: Distribution}

The completed shoe will then be packaged and shipped to the pedorthist. For custom footwear for normal feet (as contrasted with CTF for problem feet), it is likely that the manufacturer will include the insert in the shoe and ship that to the customer directly or to the retailer who has determined the customer's fit and style requirements. For CTF, it is

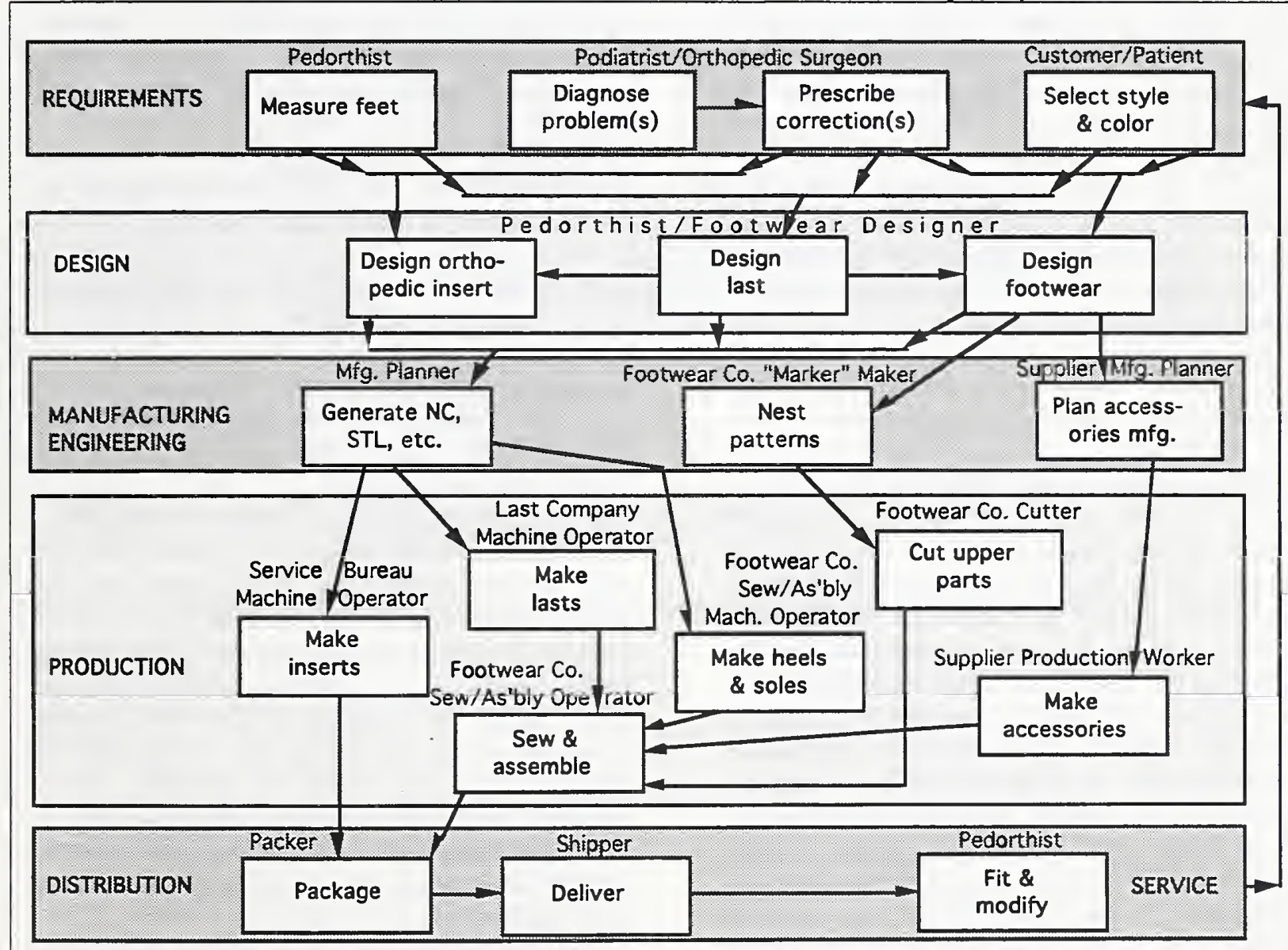

Figure 1: Custom Therapeutic Footwear Manufacturing Life Cycle

\section{4: Production}

During the production phase, all of the components are made, and the footwear is assembled more likely that the pedorthist would deal with the orthopedic insert supplier direct and receive the inserts and footwear from separate insert and footwear suppliers. 


\section{6: Service}

For CTF, the pedorthist will play a leading role as a service provider, CTF designer, and coordinator on behalf of the customer. The pedorthist will receive the footwear and inserts and do the final fitting on the customer. Small modifications or adjustments are usually required, which the pedorthist can do within the pedorthist's shop. Hence, the pedorthist closes the loop by providing final customer satisfaction to the initial requirements determined.

\section{3: Systems and Information Require- ments}

For each phase of the manufacturing life cycle, technology can enable greater productivity by transforming labor-intensive to intelligence-intensive processes. Furthermore, intelligent information exchange throughout the entire virtual enterprise can produce greater efficiency and provide the best quality, prices, and responsiveness to the ultimate consumer. The types of systems required for CTF manufacturing and the types of product information needed to communicate among those systems are shown in Figure 2: Custom Therapeutic Footwear Manufacturing Systems Overview.

The technologies anticipated for the systems shown in Figure 2 are described here. (Many of these have been or shortly will be commercialized.)

\section{1: Requirement Specification Systems}

Several types of systems are necessary to determine and specify CTF requirements and convert them to computer-interpretable form. A pedorthist will use a laser-based scanner to determine the 3D shape of a foot. The "cloud" of data points acquired by the scanner will then be processed to create a representation that is suitable for further processing by other systems. A podiatrist will use a medical diagnostic system to help determine foot problems and prescribe CTF-related corrections. That information will directly reference the $3 D$ foot measurements. The customer will then select a footwear style from a computer-based library of styles, filtered to show only appropriate selections that are relevant to the customer's feet shape and problems. Once the measurement, medical, and style requirements are determined, they will be communicated electronically to computer-aided design (CAD) systems.

\section{2: Design Systems}

Separate CAD systems (or at least separate modules of one packaged CAD system) will be used by the pedorthist and/or CTF designer to design the CTF last, insert, and footwear. The design systems will take into account the specific foot shapes, prescriptive requirements, and style selections. The last design system has the challenge to take the foot shape and the last shape desired (the style shape) and suitably integrate the two shapes to give the look of the style and accommodate the particular foot. The insert must be designed to fit into the shoe as represented by the last for the bottom of the insert and by the foot contours, suitably corrected for therapeutic corrections, for the top of the insert. (Usually, the insert is not designed for a particular last, though that consideration could be beneficial.) The footwear design system would be used to design the patterns pieces for the uppers, the heels and soles, the construction details, etc. On output, the CAD systems will generate the appropriate design specifications that are sent to the particular last, insert, and footwear manufacturer required.

\section{3: Manufacturing Engineering Systems}

Manufacturing planning systems will receive the design specifications and enable the system operators to plan the respective CTF component's manufacture. It is possible that existing systems can be used to plan much of the manufacturing.

The last is likely to be made by a numericallycontrolled machining process (such as an NC lathe or mill, suitably adapted). Consequently, the NC program for the specific machine to be used would be a major portion of the manufacturing engineering phase. Alternatively, it is possible that the orthopedic insert might be made by a rapid prototyping technique such as stereolithography. In that case, an STL-formatted file would be needed. (STL, originally an abbreviation for a data interface to stereolithography systems, is now a de facto industry standard data format that is used as an interface to most rapid prototyping systems.)

For the footwear itself, if the pattern pieces are to be cut by an NC cutter, the cutter path must be programmed to generate the maximum yield from the material used and should account for the varying grade, including defects, in different areas of the material. Determining the pattern-piece placement on the material for maximum yield is called "nesting." Other components for the footwear, such as shoe 
laces, spikes for golf shoes, etc., must also be planned in this phase and contracted out if necessary. with other shape information, modified, and ultimately transferred to the insert, last, and footwear manufacturers. In other words, each process must

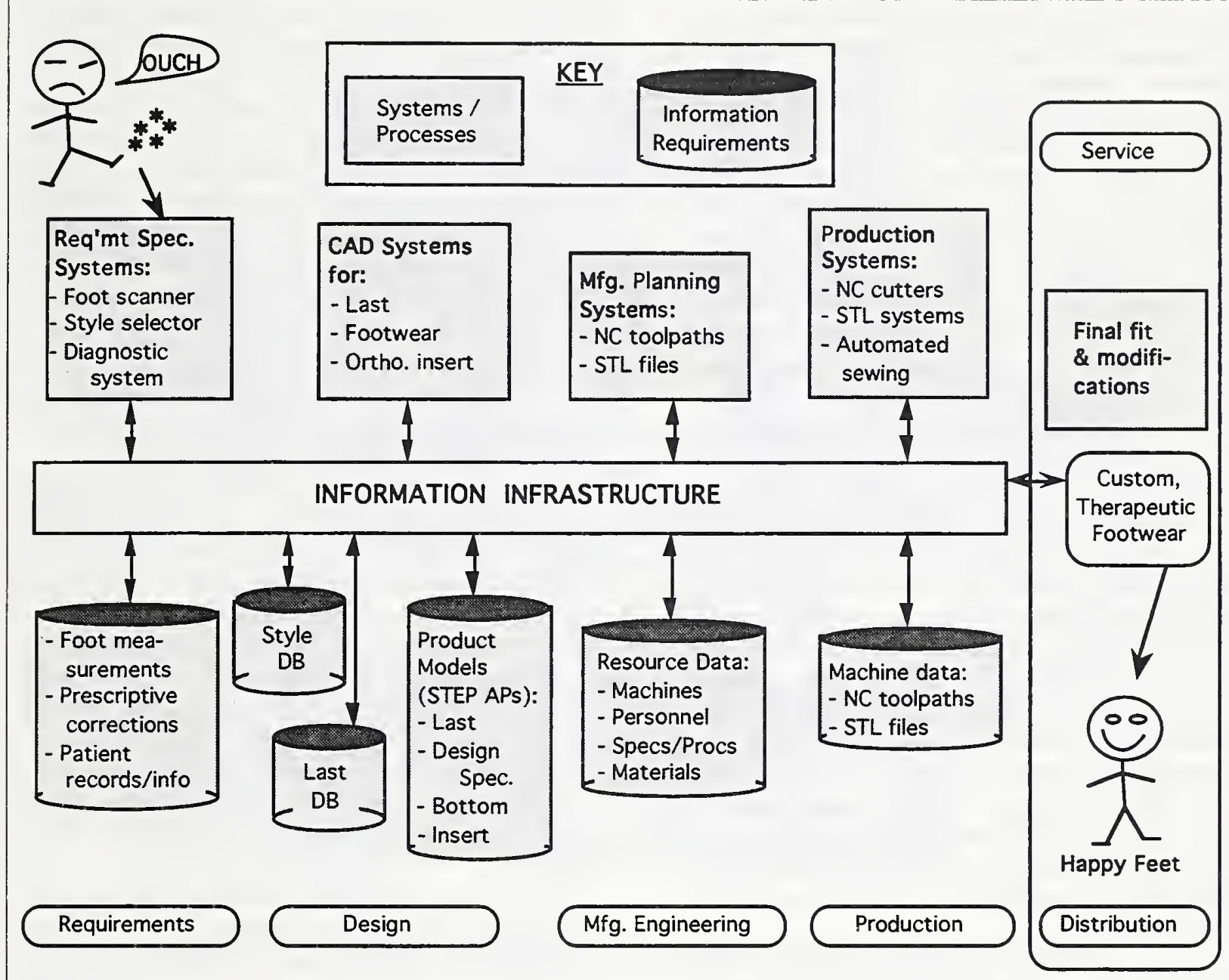

Figure 2: Custom Therapeutic Footwear Manufacturing Systems Overview

\section{4: Production, Distribution, and Service}

The files generated by the manufacturing planning systems will be sent to the automated production equipment; the lasts, patterns, and inserts will be produced; and the CTF will be assembled. After production the completed CTF and inserts will be packaged and delivered to the pedorthist. The pedorthist will do the final fitting and adjustments to produce the best fit possible for the customer.

\section{4: STEP Application Protocols}

For the CTF virtual enterprise to work, complex $3 \mathrm{D}$ shape information must be acquired, synthesized have interfaces to receive and transfer complex shape and other manufacturing information. Furthermore, those interfaces need to be carefully structured, standardized, sufficiently robust, and unambiguous to avoid the situation where interfaces are customized for particular manufacturing systems. In addition, the information interfaces that represent the foot shape, the last shape, the shoe shape, and the insert shape should be compatible with each other for the maximum information-exchange efficiency.

STEP Application Protocols (APs), that represent product information, are specifically designed to communicate manufacturing information over manufacturing-systems interfaces. In tracing the CTF manufacturing life cycle, the initial measurement, medical, and style requirements can be communicated from the "requirement-specification 
systems" to the last, footwear, and insert design systems through STEP interfaces-i.e., STEP APs.

A STEP AP that will enable the standard communication of customer requirements in terms of foot measurements and related diagnostic and prescriptive requirements for CTF would be very helpful to the industry, particularly in light of the current heavy activities focused on technology development in this part of the CTF life cycle. STEP is intended to represent product information. For CTF, STEP would consider the foot a "product" that needs to be modeled in a format consistent with the products (e.g. footwear) that will interact with the foot in order to design those products. The foot can be considered a "life-cycle product" in that it has been created, undergoes transformations over time, and can be affected, for better or for worse, by interactions with other products. Modeling the foot as a STEP AP for the purpose of designing footwear is a bridge between the medical and engineering communities-between medical requirements and manufacturing - to represent body parts in a form that can be used in product design systems for products that are interfaced to the human body.

In the design phase of the life cycle, design systems will generate design specifications, that can be represented by STEP APs, and will be communicated to the respective production facilities. At those facilities, manufacturing planning systems can read the STEP APs and generate process plans and/or toolpaths, etc. to best schedule and run the specific production equipment available at the facility to realize the designs.

For all the STEP APs developed for the CTF enterprise, existing STEP constructs can be used as appropriate. For example, since the foot, last, and inserts will be complex 3D shapes, existing STEP constructs related to complex geometries and topologies can be utilized. Other STEP integrated resources, as well as applicable STEP APs, can also be used as appropriate.

\section{5: Virtual Enterprise}

The types of companies and individuals who are likely to be interconnected in the CTF virtual enterprise are shown in Figure 3: Scenario for Custom Therapeutic Footwear Virtual Enterprise. Each shaded block represents a different company or individual. The figure represents a scenario-a particular partnership of companies to service CTF customers. (Note that the figure shows only one of the scenarios that are possible.)
As shown in the figure, a person visits a podiatrist because of a foot problem. The podiatrist diagnoses the problem and specifies a prescription for CTF. The prescription is communicated through a STEP interface to a pedorthist, who measures the foot using a foot scanner. Perhaps there needs to be some communication back and forth between the pedorthist and podiatrist so that the prescription can be referenced directly to the foot measurement, which is represented by a STEP AP. The pedorthist shows the customer a selection of footwear styles that is restricted to those that can accommodate the particular customer's feet and medical requirements. The styles are taken from a database that may include styles generated by a third-party designer.

The pedorthist then enters the style selected by the customer into a CTF design system and accesses the footwear-design specifications for that style. The design system enables the pedorthist to modify the specifications to accommodate the particular sized and shaped-foot and to add the therapeutic accommodations and corrections required. The final CTF design specification is comprised of last, insert, and footwear-design specifications. The footweardesign specification is broken down into a bottom (heel and sole), pattern (for the upper), other design specifications that include materials, construction details, accessories required (for example, shoe laces), etc. The specification for each component is sent to the appropriate manufacturer through a STEP interface.

The manufacturers make the respective components. The footwear manufacturer makes the upper, forms it on the last, and attaches the insole and bottom.

The figure was created to make the point that the separate companies involved might be very specialized. For example, it is not unlikely that a footwear manufacturer will make uppers and bottoms in the same factory. In the figure, the footwear and bottom manufacturers are shown as separate companies. Nevertheless, the scenario shown is realistic. It is not uncommon for a footwear manufacturer who makes the whole shoe to have some contracts where the uppers or bottoms are made by other contractors.

Of course, the main point made by the figure is that the CTF enterprise will be virtual, distributed, and comprised of different types of companies; and for the CTF virtual enterprise to work, product information must be communicated throughout the complex. As discussed in the previous section, STEP is ideally suited for that task. STEP APs (or portions of APs) are used to communicate the detailed design 
specifications to the separate component

key CALS technology. However, the alternative manufacturers as required.

business scenarios that are likely to coexist within the same nationwide, multi-enterprise marketplace

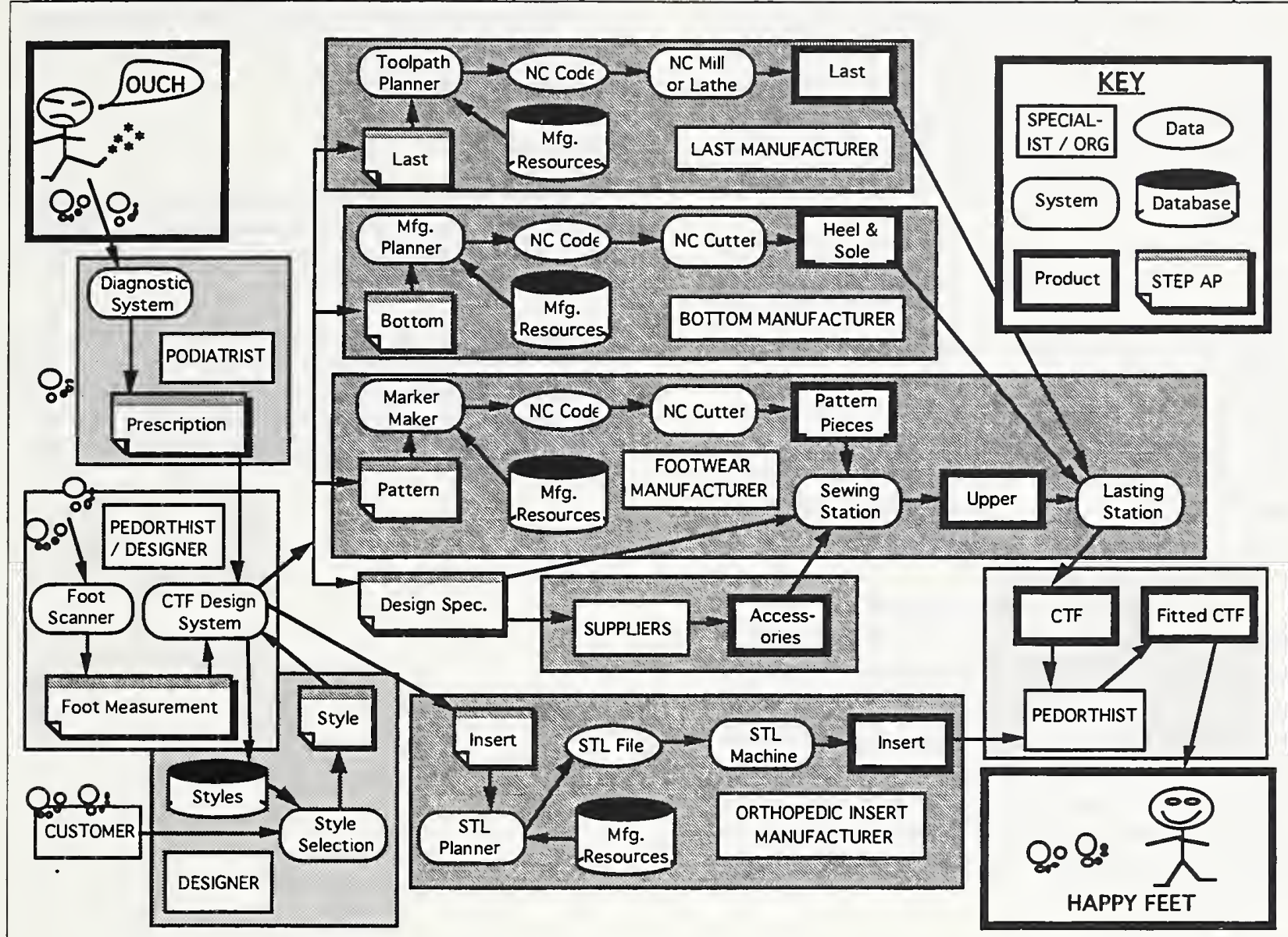

Figure 3: Scenario for Custom Therapeutic Footwear Virtual Enterprise

Once the manufacture of the shoe is completed, it is packaged and shipped to the pedorthist. The pedorthist does the final fitting on the customer and makes final adjustments as necessary.

For custom footwear for normal feet (as contrasted with CTF for problem feet), it is likely that the manufacturer will include the insert in the shoe and direct ship that to the customer or to the retailer who has determined the customer's fit and style requirements. For therapeutic footwear (CTF), it is more likely that the pedorthist would deal with the orthopedic insert supplier directly and receive the inserts and footwear from separate insert and footwear suppliers. This latter scenario, which is most likely, depends on complex 3D shape information that represents the last to be completely and unambiguously shared and used among separate organizations. This need can be satisfied by STEP, a requires that other CALS technologies be incorporated as well. For example, those related to business transactions, interface management, workflow management, and distributed data/information management are also essential.

\section{6: Summary/Conclusions}

Automation technologies that will greatly increase the productivity of the custom footwear industry are imminent. The increased productivity, leading to affordable custom-footwear prices, are likely to lead to expanded and new markets.

The new markets will depend on a virtual enterprise where geographically-distributed organizations are connected through a common information infrastructure. That infrastructure will depend on effectively communicating and managing complex 
product information and transactions among the diverse organizations involved.

CALS technologies and standards are well suited for the communications and information management required. In particular, STEP, one of the CALS standards, is very well suited to represent the complex engineering and manufacturing information that must be exchanged among the manufacturing processes distributed throughout the virtual, footwear enterprise.

In the first phase of the CTF manufacturing life cycle, a STEP AP could represent customer requirements in terms of foot measurements and related CTF prescriptions. That AP would be very helpful in light of the current substantial efforts to develop new manufacturing systems that include those requirements. Without a well-thought-out standard for this phase of the life cycle, the diverse manufacturing systems anticipated will need specialized interfaces for every process they will link to.

As a final thought, the CTF industry is likely to remain a cottage industry, albeit a greatly expanded one of small high-technology companies, because of the diverse specialists necessary, each with considerable expertise in their respective domains. Enabling their effective communication of product information, business transactions, work flow, etc. is critical for them to reap the full benefits possible to provide custom therapeutic footwear to all the people who need them.

\section{Acknowledgments}

This work is supported by the government initiative on High Performance Computing and Communications (HPCC), which is primarily being supported through the Systems Integration for Manufacturing Applications (SIMA) initiative. The work is being done by the Manufacturing Systems Integration Division of the Manufacturing Engineering Laboratory of the National Institute of Standards and Technology.

\section{Disclaimer}

No approval or endorsement of any commercial product by the National Institute of Standards and Technology is intended or implied. Certain commercial equipment, instruments, or materials are identified in this report in order to facilitate understanding. Such identification does not imply recommendation or endorsement by the National Institute of Standards and Technology, nor does it imply that the materials or equipment identified are necessarily the best available for the purpose.

This publication was prepared by United States Government employees as part of their official duties and is, therefore, a work of the U.S. Government and not subject to copyright.

\section{References}

1. Moncarz, H. T., Program Requirements to Advance the Technology of Custom Footwear Manufacturing, NISTIR 5521, National Institute of Standards and Technology, Gaithersburg, MD, October 1994.

2. Davis, Arnold S. and Research Triangle Institute, Custom Shoe Therapy, Volume 1-The Fitting of Custom Orthopedic Footwear, prepared for the National Institute of Disability and Rehabilitation Research and the National Aeronautics and Space Administration, Research Triangle Park, NC. 

